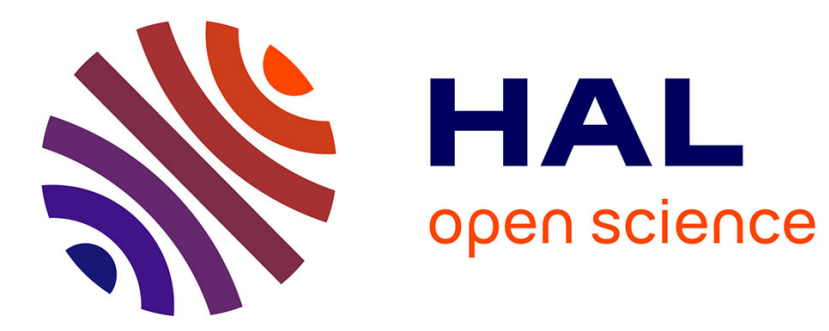

\title{
Colloidal Design of Plasmonic Sensors Based on Surface Enhanced Raman Scattering
}

Cyrille Hamon, Luis Liz-Marzán

\section{To cite this version:}

Cyrille Hamon, Luis Liz-Marzán. Colloidal Design of Plasmonic Sensors Based on Surface Enhanced Raman Scattering. Journal of Colloid and Interface Science, 2018, 512, pp.834-843. 10.1016/j.jcis.2017.10.117 . hal-02349042

\section{HAL Id: hal-02349042 \\ https://hal.science/hal-02349042}

Submitted on 5 Nov 2019

HAL is a multi-disciplinary open access archive for the deposit and dissemination of scientific research documents, whether they are published or not. The documents may come from teaching and research institutions in France or abroad, or from public or private research centers.
L'archive ouverte pluridisciplinaire HAL, est destinée au dépôt et à la diffusion de documents scientifiques de niveau recherche, publiés ou non, émanant des établissements d'enseignement et de recherche français ou étrangers, des laboratoires publics ou privés. 


\title{
Colloidal Design of Plasmonic Sensors Based on Surface Enhanced Raman Scattering
}

\author{
Cyrille Hamon ${ }^{\mathrm{a}}$, Luis M. Liz-Marzán ${ }^{\mathrm{b}, \mathrm{c}, \mathrm{d}}$
}

${ }^{a}$ Laboratoire de Physique des Solides, Univ. Paris-Sud, CNRS, UMR8502, 91405 Orsay Cedex, France.

bionanoplasmonics Laboratory, CIC biomaGUNE, Paseo de Miramón 182, 20014 Donostia - San Sebastián, Spain

'Ikerbasque, Basque Foundation for Science, 48013 Bilbao, Spain

${ }^{\mathrm{d}}$ CIBER de Bioingeniería, Biomateriales y Nanomedicina, Ciber-BBN, 20014 Donostia - San Sebastián, Spain

\begin{abstract}
This feature article focuses on the use of colloid chemistry to engineer metallic nanostructures toward application in surface enhanced Raman scattering (SERS) sensing, in particular for 'real-life' applications, where the analyte may be present in complex mixtures. We present a broad summary of the field, including recent advances that have been developed during the past 10 years. Real-life applications require a rational design and we aimed at identifying the key elements involved in it. The discussion is centered around colloidal plasmonic nanoparticles and therefore we start from the library of morphologies that have been reported in the literature. To complete the picture, colloidal self-assembly, surface chemistry and the combination with materials science techniques are highlighted. Considering the progress in the field, SERS may ultimately realize its full potential as an ultrasensitive tool for routine analytical applications.
\end{abstract}

*Email: hamon.cyrille@gmail.com (C.H.); 1lizmarzan@cicbiomagune.es (L.M.L.-M.)

Keywords: Surface enhanced Raman scattering; SERS; colloidal synthesis; plasmonics 


\section{Introduction}

Chemical sensors can be defined as devices that transform a chemical signal into analytically useful information. A chemical sensor is essentially composed of a molecular recognition element, the receptor, and a transducer that can convert a binding recognition event into a measurable signal. This definition is very general and encompasses mostly all types of chemical sensors. However, selecting a transducer or a receptor among the vast collection that has been reported in the literature is not an easy task. In this context, previous reviews classified sensors according to their figures of merit, such as sensitivity, selectivity, reproducibility, high throughput and potential use by non-experts. Electrochemical sensors, ion channel switches, fluorophores in e.g. enzyme based assays or else piezoelectric based transducers, have been critically reviewed in this way [1-3]. We aim herein at applying similar concepts to surface enhanced Raman scattering (SERS) spectroscopy, as an emerging analytical tool.

SERS was discovered in the 1970's [4, 5], but ever since the field appears to be in constant rejuvenation, partly due to the great progress in the development of well-defined colloidal plasmonic nanoparticles, which act as the transducer in a SERS-based device. A few but important points must be considered to capture the essential features of this technique. First of all, the transduction mechanism originates from the plasmonic properties of metallic nanoparticles that can confine light into nm-sized areas that feature intense electromagnetic fields, so-called hotspots, where SERS enhancement preferentially occurs. Therefore, the analyte should be localized as close as possible to the nanoparticle surface, typically at distances below $10 \mathrm{~nm}$, so that SERS can be measured, rather than conventional Raman scattering. Second, the optical read-out corresponds to the vibrational fingerprint of the analyte. Although both related to vibrational transitions, Raman scattering is significantly distinct to IR spectroscopy and we find it is worth spending a few words to explain the main differences. A first discordance can be found in the physical mechanism, as Raman scattering is based on 
inelastic scattering while IR spectroscopy is based on IR light absorption. It should also be stressed that, the information on vibrational transitions provided by both techniques is complementary, strong signals in IR spectroscopy usually being weak in Raman scattering spectroscopy and vice versa. A particularly relevant example is the strong water signal in IR spectroscopy, which is weak in Raman scattering, thereby providing us with the opportunity to readily investigate aqueous and biological samples. Interestingly, plasmonic effects can be used in IR spectroscopy, leading to so-called surface enhanced infrared absorption (SEIRA) spectroscopy, but significantly less effort has been put into this direction so far, presumably because of the poorer limit of detection (LOD), as compared to SERS. As we focus this review on SERS, we refer those readers who are interested in SEIRA, to other specialized reviews [6, 7]. Overall, SERS is promising as an analytical technique, as it provides molecule-specific information with enough sensitivity to study either a single analyte or various species simultaneously, since molecular vibrations can be readily distinguished (SERS peaks are typically narrow). Although companies such as Ocean Optics, Silmeco or Hamamatsu among other, already sells SERS substrates made of metallic nanostructures, SERS is not yet widely used as an analytical technique mainly because it suffers from certain limitations, such as issues related to signal contamination and insufficient reproducibility. Note that commercially available SERS substrates are in general prepared by physical deposition techniques but not from wet chemistry approaches. On the other hand, SERS substrates made by colloidal synthesis approaches would results in atomically smooth, well-defined surfaces, while containing tailored nanoscale features, thereby improving the measurements reproducibility. Herein, we thus restrict the discussion to colloidal approaches for SERS and provide the key elements that may help transfer new SERS substrates to the market. In this feature article, the spotlight is directed toward the importance of crossing fields such as colloid science, surface chemistry and materials science, to meet the main challenges associated to SERS detection. We 
schematically show our view on this scope through a spider-web diagram, in Figure 1. The article is structured in such a way that it presents what we consider the 5 most important points that should be considered toward addressing the major issues associated to SERS (Figure 1), namely reproducibility, simplicity, selectivity, high throughput and sensitivity. A suitable combination of these parameters should be used to optimize the overall sensing performance, as indicated by the spreading red area in Figure 1. By presenting the recent literature within this general framework, we aim not only at pinpointing diversity in SERS based technologies, but also to help building bridges between different communities, which might be key toward addressing current and future challenges.

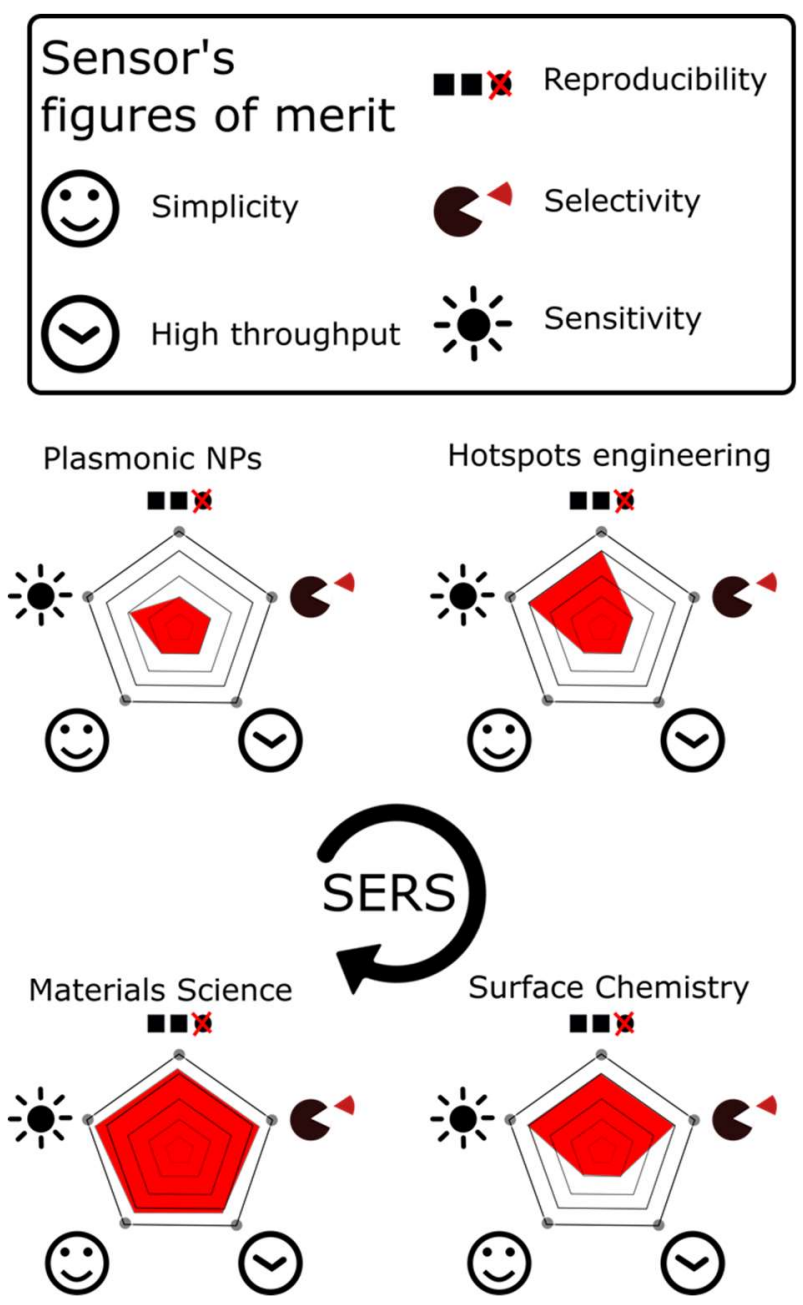

Figure 1. Spider web diagram in which the 5 edges correspond to one of the identified figures of merit of a SERS sensor. The red surface increases with the performance of the SERS sensor, by successively combining plasmonic nanoparticles, hot-spot engineering, surface chemistry 
and materials science. Plasmonic NPs as SERS transducers provide sensors with moderate sensitivity. Hotspot engineering (optimized NP morphology and directed self-assembly) results in improvement of sensitivity and reproducibility. Surface chemistry enhances selectivity, whilst materials science methods can dramatically increase throughput and facilitate operation by non-expert users.

\section{Analytical Applications of SERS}

The potential impact of SERS in various aspects of analytical chemistry is summarized in Table 1, where we collected representative examples from the literature, spanning a broad range of applications. We listed experimental parameters from selected literature reports, where different plasmonic substrates, surface chemistry and SERS setups were used for detection in complex media. A fair comparison of the SERS performance for the numerous works presented here is however not an easy task because of the wide variation in working conditions. Concentration ranges are indicated in the table, rather than LOD, because this parameter is not consistently provided in all manuscripts. Concentration ranges indicated in Table 1 may appear occasionally too high, as compared to often reported ultralow detection limits, which illustrates the difficulties to be expected when analyzing an analyte in real samples, rather than under laboratory, contaminant-free conditions. Although the information contained in each column of the table might deserve a separate review, we intend to present an overall picture of the field. In the following sections, the experimental conditions used in each example are detailed, with a focus on the current strategies toward boosting the selected figures of merit of SERS sensors (Figure 1). After a short description of the plasmonic features required for efficient SERS (Section 2), we describe methods to improve signal reproducibility through colloidal selfassembly (Section 3). We then discuss how the nanoparticle surface chemistry can affect the selectivity toward a specific analyte (Section 4), and we close the discussion by presenting 
recent advances in the use of various aspects of materials science toward SERS detection (Section 5).

Table 1. Summary of experimental parameters used in the literature for SERS detection in real samples. Examples were selected on the basis of the complexity of the medium, so that the diversity of applications in SERS based technologies is represented. Details on acronyms can be found in the corresponding references. $*$ Nanoparticles are in a solid-state material. $* *$ Nanoparticles are in colloidal form.

\begin{tabular}{|c|c|c|c|c|c|c|}
\hline $\begin{array}{c}\text { Plasmonic } \\
\text { substrate }\end{array}$ & Coating & Analyte & $\begin{array}{c}\text { Concentration } \\
\text { range }\end{array}$ & Medium & $\begin{array}{c}\text { Field of } \\
\text { application }\end{array}$ & Ref. \\
\hline $\begin{array}{c}\text { Au nanorod } \\
\text { supercrystal* }\end{array}$ & CTAB & Carbaryl & $5 \cdot 10^{-6}-1.5 \cdot 10^{-4} \mathrm{M}$ & Apple juice & Food safety & {$[8]$} \\
\hline $\begin{array}{c}\text { Au nanorod } \\
\text { supercrystal* }\end{array}$ & CTAB & Prions & $10^{-10}-10^{-6} \mathrm{M}$ & $\begin{array}{l}\text { Bovine } \\
\text { serum }\end{array}$ & $\begin{array}{c}\text { Food safety, disease } \\
\text { prevention }\end{array}$ & {$[9]$} \\
\hline Au spheres* & $\begin{array}{c}\begin{array}{c}\text { Thin } \mathrm{SiO}_{2} \\
\text { shell }\end{array} \\
\end{array}$ & $\begin{array}{c}\text { Methyl } \\
\text { parathion }\end{array}$ & trace & Fruit skin & Food safety & [10] \\
\hline $\begin{array}{c}\text { Au nanorod } \\
\text { supercrystal* }\end{array}$ & $\begin{array}{c}\text { Mesoporous } \\
\mathrm{SiO}_{2} \\
\end{array}$ & Pyocyanin & $10^{-14}-10^{-4} \mathrm{M}$ & $\begin{array}{c}\text { Bacterial } \\
\text { biofilm }\end{array}$ & Bacterial infections & [11] \\
\hline Au nanostar* & $\begin{array}{l}\text { Citrate } \\
+ \text { SDS } \\
\end{array}$ & Thiabendazole & $10^{-7}-10^{-3} \mathrm{M}$ & Fruit skin & Food safety & {$[12]$} \\
\hline $\begin{array}{c}\mathrm{Au} \\
\text { aggregates* }\end{array}$ & Citrate & PCB 77 & $3.10^{-9} \mathrm{M}$ & Soil extract & $\begin{array}{c}\text { Environmental } \\
\text { monitoring }\end{array}$ & [13] \\
\hline $\begin{array}{l}\text { AgNP 3D } \\
\text { networks* }\end{array}$ & Glucose & Melamine & $10^{-6}-10^{-3} \mathrm{M}$ & Milk & Food safety & {$[14]$} \\
\hline $\begin{array}{c}\mathrm{Au} \\
\text { aggregates** }\end{array}$ & $\begin{array}{c}\text { Citrate + } \\
\text { 4-MBA (pH } \\
\text { sensitive) }\end{array}$ & $\mathrm{pH}$ & pH: 5.4-6.8 & $\begin{array}{l}\text { Intracellular } \\
\text { environment }\end{array}$ & Diagnostics & [15] \\
\hline AgNPs** & $\begin{array}{c}\text { Citrate }+ \\
\text { glucose }\end{array}$ & Pigments & - & $\begin{array}{l}\text { Sample from } \\
\text { art piece }\end{array}$ & Art preservation & [16] \\
\hline $\begin{array}{l}\text { Ag clusters - } \\
\text { SERS Tag** }\end{array}$ & $\begin{array}{l}\text { PVP\&BSA } \\
\text { + SPDP } \\
\text { (receptor) }\end{array}$ & $\begin{array}{l}\text { Circulating } \\
\text { cancer cells }\end{array}$ & - & $\begin{array}{l}\text { Complex } \\
\text { buffer }\end{array}$ & Cancer diagnostics & {$[17]$} \\
\hline $\begin{array}{c}\mathrm{Ag} \\
\text { aggregates** }^{*}\end{array}$ & Citrate & Levofloxacin & $10^{-4}-10^{-3} \mathrm{M}$ & Treated urine & Drug monitoring & {$[18]$} \\
\hline $\begin{array}{c}\mathrm{Ag} \\
\text { aggregates** }\end{array}$ & Citrate & $\begin{array}{l}\text { Metham- } \\
\text { phetamine }\end{array}$ & $10^{-9}-10^{-3} \mathrm{M}$ & $\begin{array}{c}\text { Saliva } \\
\text { (filtered) }\end{array}$ & Drug monitoring & [19] \\
\hline Ag NPs ** & Citrate & $4-\mathrm{ABT}$ & - & Air & $\begin{array}{l}\text { Environmental } \\
\text { safety }\end{array}$ & {$[20]$} \\
\hline
\end{tabular}

\section{Plasmonic Nanoparticles as Transducers}

The intensity of the measured Raman signal is closely related to the intensity of the electromagnetic field acting on the molecule, which can be largely enhanced when the molecule is adsorbed on the surface of plasmonic nanoparticles, which therefore become an essential feature of SERS. The so-called electromagnetic enhancement in SERS originates from localized 
surface plasmon resonances (LSPR), occurring when the conduction electrons in a metal nanoparticle collectively oscillate, in resonance with the frequency of incident light. Therefore, the analyte molecule not only experiences the electromagnetic field of the laser beam, but it also interacts with the field induced by the LSPR oscillation, which is primarily concentrated at vertices, tips or edges of metal nanoparticles. Hybridization of plasmon modes (also called plasmon coupling) originates when nanoparticles are located at very short distances, which can result in 2-3 orders of magnitude stronger electromagnetic fields at interparticle gaps, as compared to individual nanoparticles [21-23]. Such localized regions of intense local field enhancement are usually termed hot spots. Even though the electromagnetic enhancement mechanism is a central element in SERS, chemical enhancement originating from specific electronic interactions, such as charge transfer between the analyte and the plasmonic transducer, may also play a role [24]. Since the first observation of the SERS effect at rough silver electrodes, a wide variety of plasmonic substrates with SERS enhancing activity have been reported, which can be prepared by either top down (e.g. lithography) or bottom up (e.g. self-assembly, colloidal synthesis) approaches [25]. We restrict the discussion here to the sufficiently wide scope of colloidal methods, in which nanoparticles are produced by wet chemistry approaches.

In practice, a close match between the laser energy and LSPRs at the nanoparticles is required to obtain a sufficiently high signal. In this regard, nanoparticles made of silver and gold ( $\mathrm{Au} / \mathrm{Ag}$ NPs) are the most frequently used transducers for SERS, because their LSPR frequencies can be tuned within the visible and near-IR (NIR), thereby matching commercially available laser sources. It should be noted that a significant body of research is currently in progress [26-29] to replace coinage metals, but few alternative materials have been identified so far, and their enhancement properties in the visible and NIR ranges are still limited [30]. The plasmonic properties of colloidal spherical Au NPs are typically characterized by a deep red color, which 
can however be modified by tuning particle size, between $3-200 \mathrm{~nm}$, leading to LSPR maxima ranging from 515 up to $750 \mathrm{~nm}[31,32]$. For large particles (above 100nm) a dephasing effect is observed that lead to higher order plasmon modes and therefore new LSPR peaks. This influences the SERS intensity that have been observed to increase from 0 to $100 \mathrm{~nm}$ and then to decrease due to the increasing significance of radiative damping[33, 34]. Another study found similar results but pointed out that the NPs were not fully comparable in terms of sphericity and crystallinity as the larger particles exhibit higher facets size and tends to be more anisotropic[35]. Moreover, the SERS performance depends on the match between the LSPR position and the excitation sources, thus the relation between the size of NPs and the SERS intensity is hard to disentangled as the LSPR is shifted for the different NPs batches. In the case of small spherical Ag NPs, the LSPR occurs at higher energies around $400 \mathrm{~nm}$, but can also be tuned by increasing particle size [36]. Similarly to Au NPs, the relation between the Ag NPs size and SERS signal intensity have been investigated and particles diameters around 55-70 nm gave the best performances for excitation wavelengths at 532, 633 and $785 \mathrm{~nm}[34]$. Nano-alloys made of gold and silver have also been prepared by co-reduction of the corresponding metallic salts in solution, thereby shifting the plasmon resonances within the range between 380 and 520 nm [37]. For applications that require a wider spectral tunability, alternative strategies have been developed to control not only the size but also the shape of the nanoparticles. For instance, LSPRs can be considerably red-shifted by increasing the anisotropy (aspect ratio) of gold nanorods, even reaching the IR range. In a recent example, concepts borrowed from controlled living polymerization were successfully applied to the synthesis of bimetallic long $\mathrm{Au@Ag}$ nanowires, which can accommodate multiple plasmon resonance modes, spanning from the visible up to the mid-IR [38]. In this work, the authors showed that the silver dominated the optical properties in the near field with the gold core playing a marginal role. The selection of gold or silver nanoparticles for SERS is usually tailored to the targeted application, as both 
metals display excellent performance, albeit with some differences. A major parameter to be considered is the chemical stability, which is significantly higher in gold, thereby affecting the interaction between the NP surface and the analyte molecules. On the other hand, silver often yields higher enhancement factors, in particular at low wavelengths. The better plasmonic performance of silver is related to the metal interband transitions being restricted to the UV range, so that they do not interfere with plasmon resonances. Note that protecting the silver nanoparticle with a gold layer is possible in order to combine the high optical properties of silver particles with an improved chemical stability[39-41]. Practical aspects must also be considered, as the synthesis of gold nanoparticles has been described in more detail, so that a wider library of particle sizes and morphologies are available. Although spherical NPs have been most widely studied for SERS, anisotropic NPs possessing sharp tips and edges are known to display higher near field and SERS enhancements [42]. The selection of NP morphologies for SERS typically relies on the corresponding LSPR wavelength and field enhancement distribution. Recent development of electron microscopy methods has opened the possibility to characterize hot spots in situ, on both single particles and assemblies, by measuring either electron energy loss spectroscopy (EELS), or emitted photons having energy in the visible, by cathodoluminescence $[43,44]$. Since the first application of EELS to map surface plasmon resonances on silver nanotriangles [45], a wide variety of shapes have been investigated, as exemplified in Figure 2. 

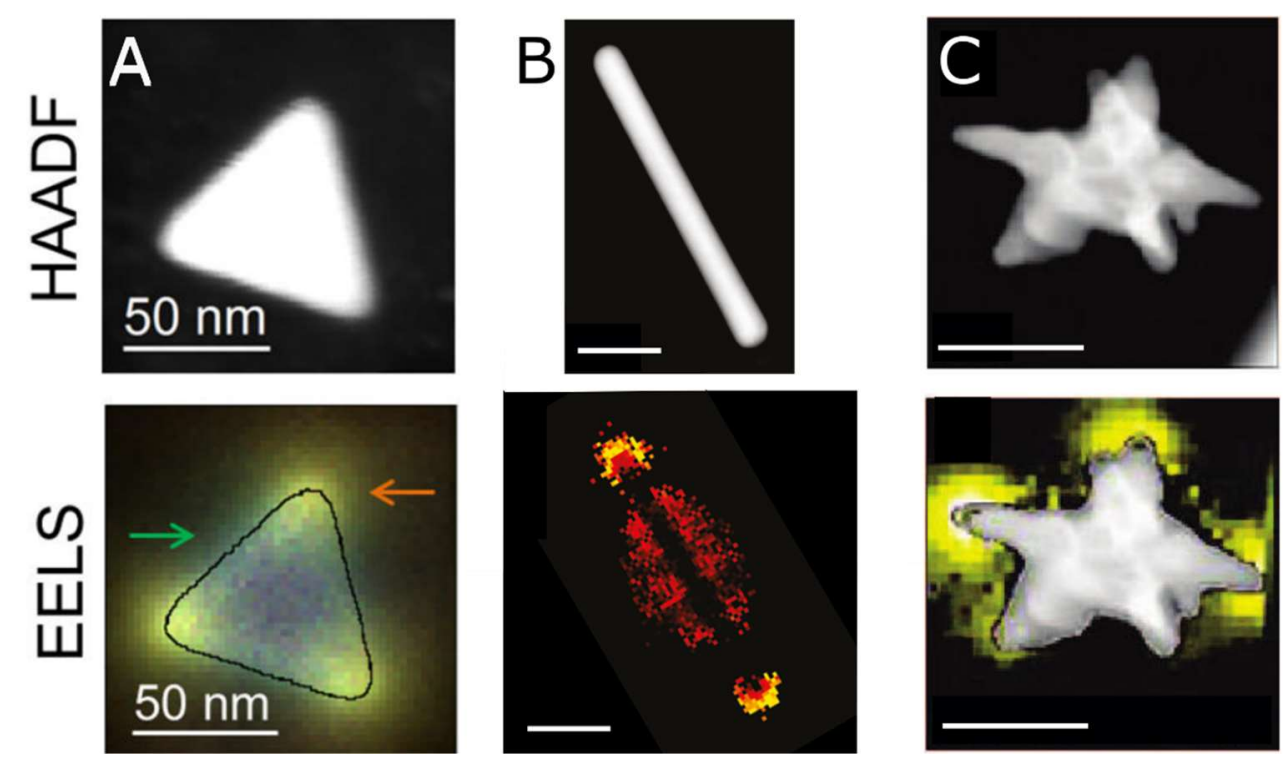

Figure 2: Plasmonic nanoparticles concentrate light at their apexes. High angle annular dark field scanning transmission electron microscopy (HAADF-STEM) images (upper panel) of plasmonic nanoparticles and corresponding EELS maps (lower panel). Colors are arbitrary, but brightness indicates areas of higher field enhancement. A) Gold nanotriangle [46]; B) Silver nanorod [47]; C) Gold nanostar [48]. Scale bars on all images are $50 \mathrm{~nm}$. A), B) and C) were adapted with permission from references [40], [41] and [42], respectively. Copyright American Chemical Society.

Since NP shape determines light confinement at the nanoscale, it also makes a strong impact in SERS applications, but it is difficult to conclude which NP type prevails. It can be seen in Figure 2 that, only a small fraction of the NP surface displays high field enhancements, where the SERS effect is produced [49]. There seems to be general agreement that multi-branched nanoparticles, also known as nanostars, are the brightest nanoparticles reported so far; many works pinpointed their high performance for SERS [50-52]. As an example of practical application, the detection of the pesticide thiabendazole has been reported down to $20 \mathrm{ppb}$ on fruit skin, using nanostars immobilized on a flexible and transparent polymer substrate [12]. Nanostars can be produced by means of seed-mediated growth methods, in which spherical nanoparticles are rapidly injected in a growth solution containing both metal salt and shapedirecting adjuvants $[53,54]$. Generally speaking, the synthesis of anisotropic nanoparticles 
requires a certain degree of control over the rate at which atoms are deposited on certain crystallographic facets of the seeds $[55,56]$. In the case of nanostars, the deposition is largely governed by reaction kinetics, resulting in a dispersion of branched nanoparticles with a rather wide variety of branch length and sharpness, even within individual particles. As a result of the low thermodynamic stability, nanostars can spontaneously reshape into spheroidal particles and therefore a suitable capping molecule or coating shell are required upon synthesis. Usual capping agents are poly(N-vinylpyrrolidone) (PVP), cetyltrimethylammonium bromide (CTAB) or thiolated molecules, while coating with silica has also been demonstrated. Unfortunately, the coating process may hinder adsorption of analyte molecules on the metal surface, in turn compromising SERS performance. Thus, a balance must be found when working with nanostars, between long term colloidal stability and SERS performance, which can be solved by cleaning the surface (removing capping ligands) prior to the analysis. As an alternative, other plasmonic transducers can be used, which may not be as efficient toward electromagnetic enhancement but display much better long-term stability, size monodispersity and ease of synthesis. Another popular choice are gold nanotriangles, which are significantly more stable against reshaping, with SERS enhancement factors in solution (from individual particles) up to $10^{5}$ [57]. Even though the synthetic protocols usually result in moderate shape yield, purification can be successfully applied to remove byproducts (particles with other morphologies), so that the content of nanotriangles can be increased above $90 \%$.

Other nanoparticle morphologies have been studied for SERS applications, such as nanobipyramids [49], porous nanoparticles [58-60] and nanoparticles featuring internal nanometer-scale gaps [61-66], though practical applications are yet to be demonstrated. More frequently used are gold nanorods, which can be readily produced in high yield $(>90 \%)$ and a variety of protocols have been developed for their preparation, with fine tuning of both particle size and aspect ratio, by simply varying a few synthesis parameters [67-69]. However, their 
SERS performance at the single nanoparticle level is inferior to that from nanoparticles with sharper tips. A detailed theoretical study has been recently reported, aiming at a fair comparison between the three most frequently used NP shapes for SERS, i.e. nanospheres, nanorods and nanostars [70]. The computational results concluded that nanostars provide the best performance at the single particle level, but nanorods outperform other shapes when assembled in dense monolayers. For further reading, a detailed analysis on the plasmonic properties of anisotropic nanoparticles can be found in a recent review by our group [42]. This discussion serves us to introduce the following section, which focuses on hot spot engineering, as a result of collective plasmonic effects.

\section{Improving SERS Detection Through Self-Assembly}

Despite of the wide variety of nanoparticles reported so far, gold and silver nanospheres are still most frequently used toward 'real-life' SERS applications (see Table 1), which stems from a trade-off toward simplicity at the expense of sensitivity. Although plasmonic nanospheres are not particularly SERS efficient at the single particle level, their performance can be greatly increased within assemblies. In practice, the aggregation of nanospheres can be readily induced by addition of charge screening agents (e.g. electrolytes), so that analyte molecules can be trapped at the resulting hotspots within the aggregates. By means of this simple process, the

drug levofloxacin has been quantified in urine samples at concentrations down to $4.5 .10^{-4} \mathrm{M}$ which is highly relevant to monitor clearance rate. In certain cases, the analyte alone can induce the aggregation of the nanoparticles, which has been demonstrated for the volatile compounds 4-aminobenzenethiol and 2,4-dinitrotoluene, which can be detected from the gas phase at trace levels $(1 \mathrm{ppb})$. However, uncontrolled aggregation often leads to a wide distribution of areas with different enhancement factors, thereby hindering quantification of the SERS signals. Within this framework, a vast amount of work has been carried out to improve signal reliability by directed self-assembly strategies (Figure 3) [71-77]. 


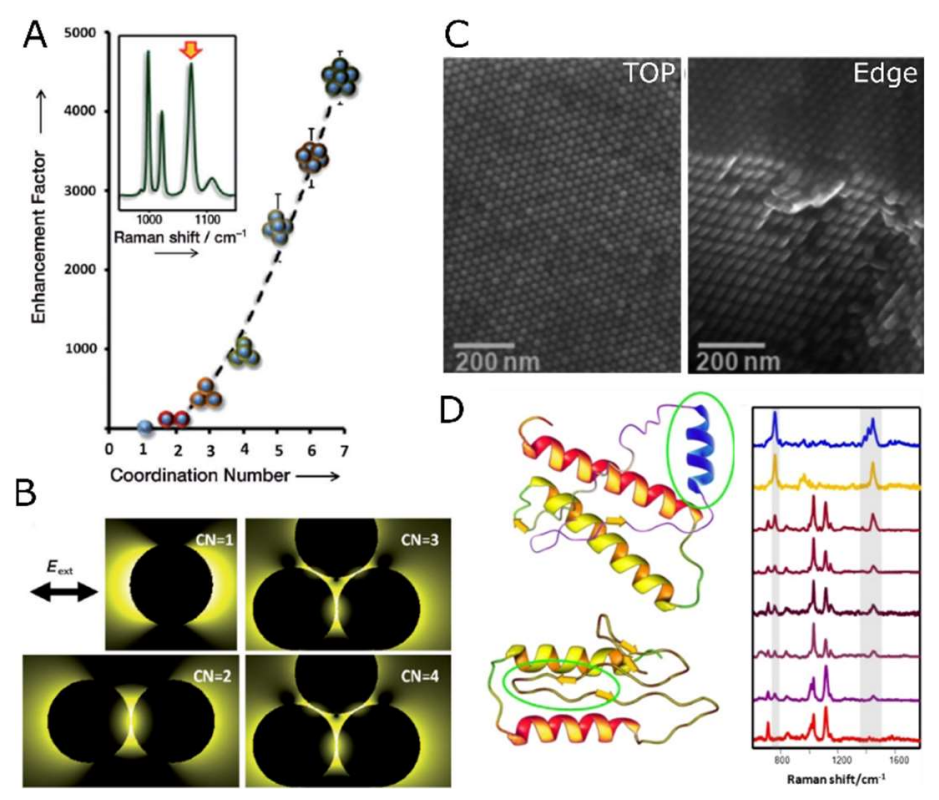

Figure 3: Self-assembly of plasmonic nanoparticles for SERS. A-B) Comparison of the SERS performance of colloidal clusters made of gold spheres; EF increases with coordination number [66]. B) Calculated near field intensity maps for four types of clusters, along the plane through the particle centers, showing intense plasmonic hot spots at interparticle gaps [66]. C) SEM images of a gold nanorod supercrystal, viewed from the top and from the edge [9]. D) SERS detection of scrambled prions at various concentrations in bovine serum, using gold nanorod supercrystals as the transducer [9]. A,B were adapted with permission from reference [66]. C,D were adapted with permission from reference [9]. Copyright 2011 National Academy of Sciences.

Self-assembly in solution or at interfaces (i.e. solid/liquid, liquid/liquid) are mainstream approaches toward the preparation of SERS substrates. This is represented on Table 1 wherein the nanoparticles are either in a solid-state material or dispersed in colloidal suspension. The design of the SERS transducer is related to the goal of the study. For instance, a solid form can be used in harsh conditions (e.g. high ionic strength) whereas a colloidal form is particularly suited to probe various region of a sample (e.g. cells internalization). Colloidal nanoparticle clusters with controlled size (aggregation number) can be prepared by means of association with block copolymers [78] or DNA origami [79-81], among other approaches [64, 82-87]. An appealing feature of colloidal plasmonic structures is the opportunity to interact with their environment, whereby the building blocks can be encoded with SERS tags [88, 89], but also 
associated with luminescent or magnetic nanoparticles, leading to multi-functional clusters [83, 90-92]. For instance, the co-encapsulation of gold nanostars and iron-oxide nanoparticles resulted in the formation of hybrid colloidal composites that were highly responsive to both light and an external magnetic field [91]. Limits of detection around $10^{-9} \mathrm{M}$ for model analytes were demonstrated upon magnetic accumulation, while the relatively small size of the clusters renders them an appealing system for bioimaging applications. On the other hand, the size of the plasmonic structure is no longer a limit when immobilized at an interface, i.e. the density of hot-spots can be dramatically increased, even in three dimensions. Monodisperse building blocks such as polyhedral $[93,94]$ and anisotropic nanoparticles [95], are required to achieve uniform nanostructures, which usually show excellent SERS performance. In line with this, arrays of standing gold nanorods have been shown to be ideal substrates, as they display a homogeneous distribution of hotspots with very high density, thereby ensuring both reproducibility and sensitivity [96]. Highly uniform SERS signals were measured by scanning the surface of organized nanorod supercrystals, and enhancement factors as high as $10^{7}$ were obtained [97]. Self-assembled gold nanorods have also been used for the detection of the pesticide carbaryl in the ppm regime, in apple juice [8]. In another example, scrambled prions dispersed in bovine serum were detected down to $0.1 \mathrm{nM}$ concentrations, even from whole blood, thus showing great promise for early diagnosis of neurodegenerative diseases [9]. The various limits of detection reported herein are not only influenced by the chemical nature of the analyte, but also by the complexity of the surrounding medium, which often requires tailored surface chemistry.

\section{The Relevance of Surface Chemistry}

The spotlight of this review has been so far directed toward the metal core of plasmonic nanoparticles. This section however focuses on their surface chemistry, which can be equally important when dealing with SERS applications. First of all, the molecular structure of the 
analyte must be taken into consideration at the time of selecting a SERS substrate. On one hand, detection can be optimized if an electronic absorption band of the analyte coincides with the energy of the exciting laser light, resulting in resonance Raman scattering, which in the presence of a plasmonic transducer leads to surface enhanced resonance Raman scattering (SERRS) [98]. On the other hand, the Raman cross section of the analyte is another intrinsic feature to be considered [99]. In general, heterocyclic molecules are characterized with the highest Raman cross sections and outperform other molecules like water or fatty acids. Considering the latter, it is not surprising that pesticides [100], various drugs [101], or dye pigments [102], which feature aromatic rings, are usual analytes of choice for SERS demonstrations. It is however more challenging to measure analytes featuring small Raman cross sections or those which do not even display relevant vibrations (e.g. metal ions), for which specific strategies have been devised [103]. In the example of ions, detection can be achieved by indirect SERS, i.e. using a Raman active ligand that can undergo a certain vibrational change upon exposure to the analyte [104]. This strategy has been used to quantify few ppb of mercury [105] and picomolar concentrations of chloride [106], in fresh aqueous samples (Figure 4a). However, demonstration in 'real-life' water samples was not shown, presumably because the presence of competitive atomic species would require the use of more specific molecular receptors. An alternative method consists of encoding nanoparticles with a Raman reporter molecule (SERS tag), as well as a biocompatible coating that can be functionalized toward a specific receptor [107, 108]. This approach may be used to detect analytes with low Raman cross section, contamination issues from the transducer are overcome, and multiplexing is possible by the simultaneous use of different SERS tags, so that various analytes can be targeted for spectroscopic biological detection or imaging applications [88, 109-114]. SERS tags that do not carry a protective layer are capable of probing both the position of the nanoparticle (imaging) and the local environment [114]. For instance, $\mathrm{pH}$ has been measured by SERS within living 
cells, employing a $\mathrm{pH}$ sensitive Raman reporter, which is highly relevant to probe $\mathrm{pH}$ in the different cell compartments (Figure 4b) $[15,115]$. A second type of SERS tags features a protective coating, such as polyethylene glycol or inorganic shells [107]. Whereas the coating hinders interaction of SERS reporters with their surroundings, it can enhance cellular uptake and colloidal stability, thereby making them particularly suited for imaging purposes. In a representative example, five different silica-coated SERS tags were spectrally separated and located in vivo after accumulation in the liver of a mouse [110]. Further discussion on applications of SERS tags in bionanalysis is beyond the scope of this feature article and we direct the reader to recent reviews $[88,107]$.
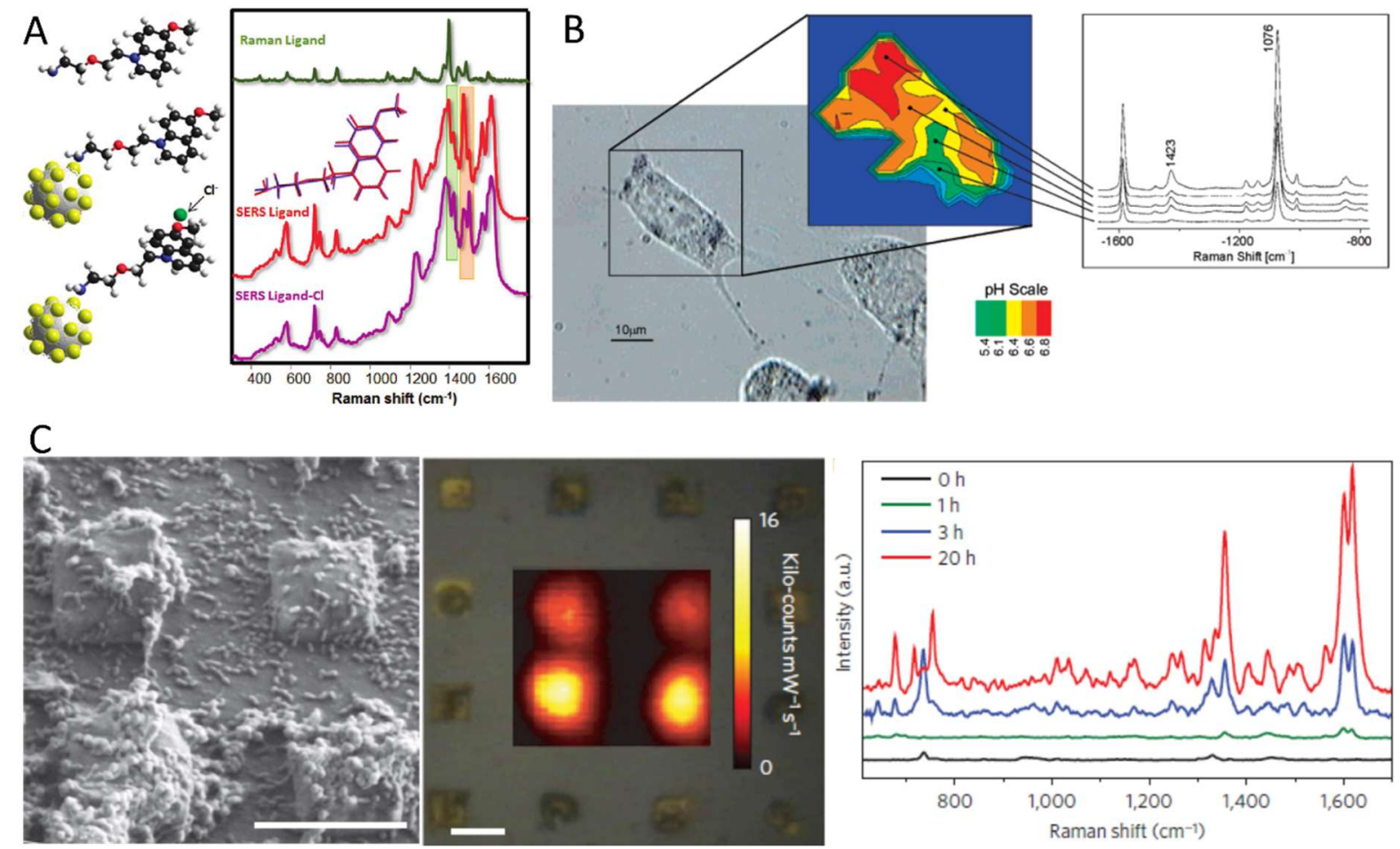

Figure 4: SERS studies adapting chemistry of transducer and receptor. A) Schematic representation of the detection of chloride by a Raman active ligand immobilized on silvercoated silica microbeads [91]. B) Probing and imaging $\mathrm{pH}$ inside individual live cells by a SERS nanosensor comprising a pH sensitive Raman reporter [15]. C) In situ detection and imaging of pyocyanin produced by Pseudomonas aeruginosa, grown on micropatterned gold nanorod supercrystals covered with mesoporous $\mathrm{SiO}_{2}$ (scale bars: $5 \mu \mathrm{m}$ ) [11]. A,B were adapted with 
permission from references [91] and [15], respectively. Copyright American Chemical Society. $\mathrm{C}$ was adapted with permission from reference [11].

The surface chemistry of nanoparticles may be modified to improve selectivity and facilitate ultradetection, in particular when the analyte is present at very low concentration in a medium containing other molecular species. In practice, there is a competition for space between the surface ligands, the analyte and potential contaminants, which must be balanced and generally results in a bottleneck when aiming at detecting very low analyte concentrations. Adsorption of the analyte on the plasmonic transducer is crucial and various issues must be addressed in this respect. It has been reported that SERS performance is enhanced when electrostatic repulsion forces between plasmonic particles and analyte molecules are minimized [116, 117]. Similarly, hydrophobic/hydrophilic interactions or the molecular affinity between the analyte and the metal surface must be considered. For instance, the SERS signal of cytochrome $\mathrm{C}$ was found to depend on the charge of the plasmonic substrate, such that positively or negatively charged surfaces lead to different protein orientations [118]. Therefore, a compromise between avoiding contamination and promoting the diffusion of the analyte toward the plasmonic surface should be achieved. In this context, a recently reported strategy comprises the use of substrates made of gold nanorod supercrystals embedded in a mesoporous silica matrix, which acts as a molecular sieve that suppresses molecular diffusion of large (bio)molecules, while allowing the penetration of small analytes within the supercrystal [119]. Interestingly, it was demonstrated that such substrates can be applied to the detection of bacterial quorum sensing metabolites, at trace concentration level (below $10^{-10} \mathrm{M}$ ), within a growing biofilm of Pseudomonas aeruginosa (Figure 4c) [11]. Even though other small molecules can also diffuse into the mesochannels, the chemical fingerprint of the analyte was clearly recorded in SERRS because pyocyanin displays an absorption band in the near-IR (resonant with a $785 \mathrm{~nm}$ laser), as well as intrinsically high Raman cross section. Other coatings can be used such as mesoporous titania, hydrogels or metal organic frameworks, which would additionally provide different 
means to tune the pore size $[11,120,121]$. Alternative approaches aim at avoiding biofouling by coating the nanoparticles with e.g. polyethyleneglycol or proteins. A recent report showed the hierarchical modification of the plasmonic transducer by a combination of a short probing ligand and a longer anti-fouling ligand, to detect doxorubicin spiked in whole blood at the micromolar range [122]. It should be noted that each application requires a different design and additional examples can be found in recent reviews focusing on the advances of SERS for in vivo diagnosis [107] and food safety [100].

\section{Crossing Borders with Materials Science}

Practical applications require a device that displays excellent performance, eventually allowing high throughput screening, while maintaining simplicity [123]. We describe in this section some opportunities that materials science can offer toward high throughput, reliability and eventually increased sensitivity. Elastomeric stamps prepared by soft lithography can be used in tandem with self-assembly to improve the deposition of nanoparticles on surfaces $[124,125]$, as recently demonstrated through the deposition of gold nanorod arrays over centimeter length scales, which offers the opportunity to tune electromagnetic enhancement at interparticle gaps, with nanoscale precision [126]. Larger supercrystals can be obtained by means of this strategy, but using templates with features that are much larger than the dimension of the nanoparticles, resulting in identical multiple copies over large areas $[127,128]$. The resulting hierarchical organization was shown to improve signal reliability over the substrate, as compared to dropcasted substrates [127].

When dealing with SERS detection in 'real-life' samples, various treatments are usually needed prior the analysis, to remove interfering species or to concentrate the analytes, which hinder the widespread use of SERS. In this respect, materials science can help simplifying those steps, by using only benchtop equipment while saving processing time. As an example, superhydrophobic surfaces can be used to concentrate an evaporating aqueous dispersion directly 
onto the plasmonic substrate [129]. This concept has been further extended using so-called omniphobic surfaces, resulting in the development of a technique that is known as slippery liquid-infused porous surface-enhanced Raman scattering (SLIPSERS) [13]. These approaches present the advantage of directly concentrating the analyte within the sensor, thereby requiring minimum user intervention. The high collection efficiency of SLIPSERS led to LOD down to attomolar concentrations, for a model analyte dispersed in ethanol, and down to $1 \mathrm{ppb}$ for polychlorinated biphenyls (PCBs) extracted from contaminated soils [13]. Other substrates such as paper can be combined with plasmonic nanoparticles toward flexible and low cost devices [130].
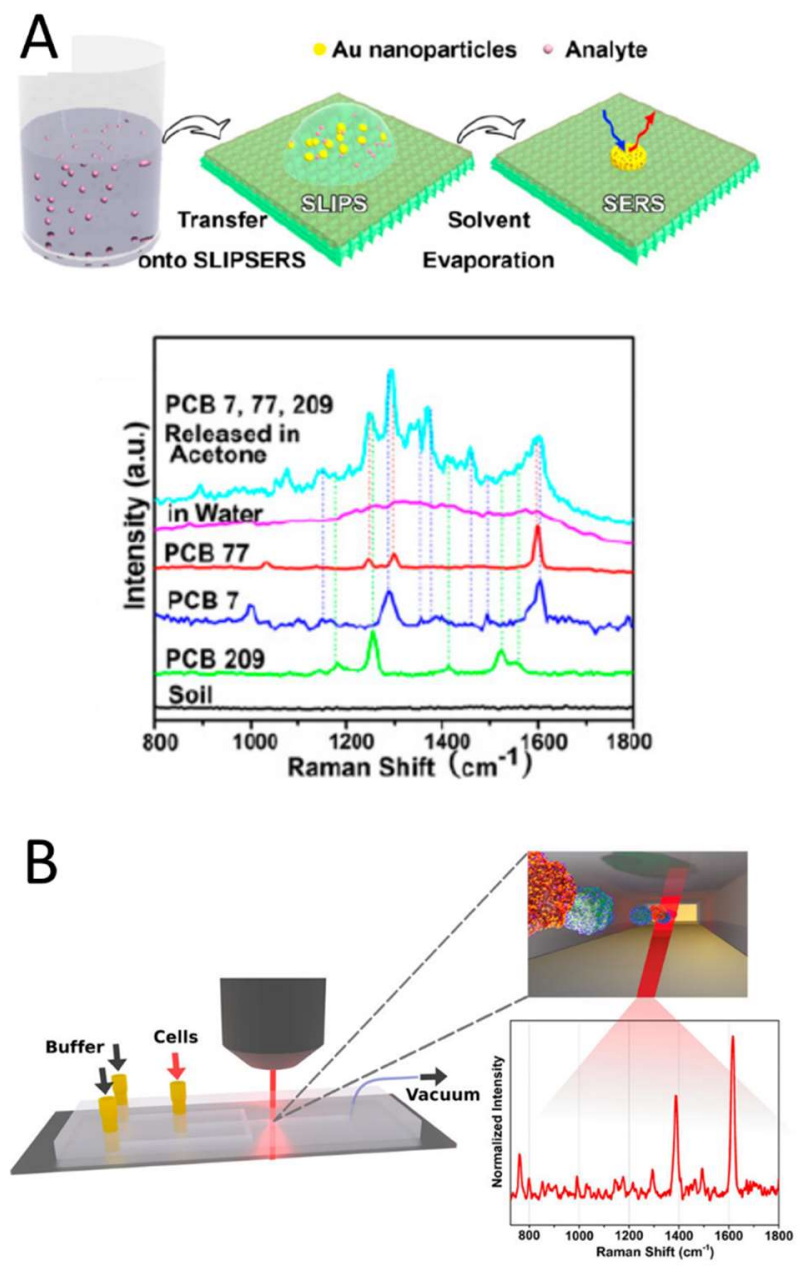

Figure 5: SERS assisted by materials science. A) Working principle of SLIPSERS and detection of PCBs extracted from contaminated soils [13]. B) Microfluidic setup used to detect 
circulating cancer cells, pre-labeled with SERS tags [17]. A was adapted with permission from reference [13]; Copyright 2015 National Academy of Sciences. B was adapted with permission from reference [17]; Copyright 2015 American Chemical Society.

Microfluidics deals with the manipulation of small volumes into microchannels and holds great promise toward numerous applications, when combined with SERS [131, 132]. Advantages include enhanced mass transport, low dosage and high throughput, as compared to conventional SERS systems. Care must be taken during the design of the sensor, such as the functionalization of the microchannel to avoid accumulation of nanoparticles within the SERS interrogation region [132], but properly designed systems have shown outstanding performance. For example, micromixers have been used to enhance the interaction between the analyte and the transducer, performing the detection of the pesticide Methyl Parathion in aqueous samples, down to $0.1 \mathrm{ppm}$ [133]. On a different application field, circulating cancer cells at low concentrations were quantified by labeling with SERS tags and flowing them through a microfluidic chip, which opens new avenues toward continuous single-cell SERS analysis [17]. Similarly, bacterial strains have been identified with dramatically lowered analysis time, when SERS was combined with microfluidics, as compared to standard SERS (4h vs. 31h) [134].

\section{Summary and Outlook}

Although a large amount of research has been carried out in the field of SERS, ranging from nanoparticle synthesis through demonstrated sensing applications, the majority of this work has still been dedicated to proof-of-concept studies. During the last decade, 'real-life' analytical applications have also been demonstrated, which may result in a clear advantage, as compared to state of the art techniques. This feature article intended to demonstrate recent advances toward 'real-life' applications of SERS, by highlighting the need for a rational design in order to to improve sensitivity, reproducibility, selectivity, simplicity and high throughput of SERS- 
based devices. Overall, the advances presented in this review show that we are close to kickoff SERS into the real world and how this can be assisted by materials science technologies. Some remaining limitations must however be resolved, before being able to purchase operating SERS kits commercially. Despite the eventual portability of microfluidic technologies, the SERS analysis of 'real-life' samples is usually carried out with bulky micro-Raman setups. Demonstration of similar performance using portable Raman spectrometers should open new avenues toward applications requiring deployable systems. Moreover, microfluidics not only require a chip but also external equipment, which so far limits the applications of SERSmicrofluidic devices to the laboratory. To ultimately obtain a device that can be used by nonexperts, it is important to minimize the bulkiness and cost of the equipment as much as possible, while keeping the performance of the device intact. Considering these limitations, various strategies have been proposed to implement pump-free microfluidics, by means of pumping lids [135] or vacuum driven flows [136]. Such advances are available to simplify the use of SERS devices in the near future. An example of application is the separation of red blood cells from whole blood and the detection of biomarkers in "self-powered" chips [137]. We envision that similar concepts can be applied to SERS and make a large impact in the field.

\section{Acknowledgements}

This work was funded by the Spanish MINECO (grant \# MAT2013-46101) and the European Research Council ((ERC Advanced Grant \# 267867, Plasmaquo).

\section{References}

[1] C.I.L. Justino, T.A. Rocha-Santos, A.C. Duarte, Trends Anal. Chem. 29 (2010) 1172-1183.

[2] C. McDonagh, C.S. Burke, B.D. MacCraith, Chem. Rev. 108 (2008) 400-422.

[3] V. Andreu, Y. Picó, Trends Anal. Chem. 23 (2004) 772-789.

[4] M. Fleischmann, P.J. Hendra, A.J. McQuillan, Chem. Phys. Lett. 26 (1974) 163-166.

[5] D.L. Jeanmaire, R.P. Van Duyne, J. Electroanal. Chem. Interfacial Electrochem. 84 (1977) $1-20$. 
[6] S. Abalde-Cela, S. Carregal-Romero, J.P. Coelho, A. Guerrero-Martínez, Adv. Colloid Interface Sci. 233 (2016) 255-270.

[7] P.R. Griffiths, Spectroscopic Properties of Inorganic and Organometallic Compounds: Techniques, Materials and Applications, Volume 44, The Royal Society of Chemistry 2013, pp. 95-122.

[8] Z. Zhang, Q. Yu, H. Li, A. Mustapha, M. Lin, J. Food Sci. 80 (2015) N450-N458.

[9] R.A. Alvarez-Puebla, A. Agarwal, P. Manna, B.P. Khanal, P. Aldeanueva-Potel, E. CarbóArgibay, N. Pazos-Pérez, L. Vigderman, E.R. Zubarev, N.A. Kotov, L.M. Liz-Marzán, Proc. Natl. Acad. Sci. U.S.A. 108 (2011) 8157-8161.

[10] J.F. Li, Y.F. Huang, Y. Ding, Z.L. Yang, S.B. Li, X.S. Zhou, F.R. Fan, W. Zhang, Z.Y. Zhou, Y. WuDe, B. Ren, Z.L. Wang, Z.Q. Tian, Nature 464 (2010) 392-395.

[11] G. Bodelon, V. Montes-Garcia, V. Lopez-Puente, E.H. Hill, C. Hamon, M.N. Sanz-Ortiz, S. Rodal-Cedeira, C. Costas, S. Celiksoy, I. Perez-Juste, L. Scarabelli, A. La Porta, J. PerezJuste, I. Pastoriza-Santos, L.M. Liz-Marzán, Nat. Mater. 15 (2016) 1203-1211.

[12] A. Shiohara, J. Langer, L. Polavarapu, L.M. Liz-Marzán, Nanoscale 6 (2014) 9817-9823.

[13] S. Yang, X. Dai, B.B. Stogin, T.-S. Wong, Proc. Natl. Acad. Sci. U.S.A. 113 (2016) 268273.

[14] N. Zhou, Q. Zhou, G. Meng, Z. Huang, Y. Ke, J. Liu, N. Wu, ACS Sensors, 10 (2016) 1193-1197.

[15] J. Kneipp, H. Kneipp, B. Wittig, K. Kneipp, Nano Lett. 7 (2007) 2819-2823.

[16] M. Leona, Proc. Natl. Acad. Sci. U.S.A. 106 (2009) 14757-14762.

[17] A. Pallaoro, M.R. Hoonejani, G.B. Braun, C.D. Meinhart, M. Moskovits, ACS Nano, 9 (2015) 4328-4336

[18] I.J. Hidi, M. Jahn, M.W. Pletz, K. Weber, D. Cialla-May, J. Popp, J. Phys. Chem C 120 (2016) 20613-20623.

[19] C. Andreou, M.R. Hoonejani, M.R. Barmi, M. Moskovits, C.D. Meinhart, ACS Nano 7 (2013) 7157-7164.

[20] B.D. Piorek, S.J. Lee, J.G. Santiago, M. Moskovits, S. Banerjee, C.D. Meinhart, Proc. Natl. Acad. Sci. U.S.A. 104 (2007) 18898-18901.

[21] G. Haberfehlner, A. Trügler, F.P. Schmidt, A. Hörl, F. Hofer, U. Hohenester, G. Kothleitner, Nano Lett. 15 (2015) 7726-7730.

[22] G. Baffou, R. Quidant, Chem. Rev. 43 (2014) 3898-3907.

[23] R. Alvarez-Puebla, L.M. Liz-Marzán, F.J. García de Abajo, J. Phys. Chem. Lett. 1 (2010) 2428-2434.

[24] S. Schlücker, Angew. Chem. Int. Ed. 53 (2014) 4756-4795.

[25] M. Jahn, S. Patze, I.J. Hidi, R. Knipper, A.I. Radu, A. Muhlig, S. Yuksel, V. Peksa, K. Weber, T. Mayerhofer, D. Cialla-May, J. Popp, Analyst 141 (2016) 756-793. 
[26] A. Comin, L. Manna, Chem. Soc. Rev. 43 (2014) 3957-3975.

[27] M.M.Y.A. Alsaif, K. Latham, M.R. Field, D.D. Yao, N.V. Medehkar, G.A. Beane, R.B. Kaner, S.P. Russo, J.Z. Ou, K. Kalantar-Zadeh, Adv. Mat. 26 (2014) 3931-3937.

[28] K. Manthiram, A.P. Alivisatos, J. Am. Chem. Soc. 134 (2012) 3995-3998.

[29] M.M.Y.A. Alsaif, M.R. Field, T. Daeneke, A.F. Chrimes, W. Zhang, B.J. Carey, K.J. Berean, S. Walia, J. van Embden, B. Zhang, K. Latham, K. Kalantar-zadeh, J.Z. Ou, ACS Appl. Mater. Interfaces 8 (2016) 3482-3493.

[30] A. Lalisse, G. Tessier, J. Plain, G. Baffou, Sci. Rep. 6 (2016) 38647.

[31] M. Brust, M. Walker, D. Bethell, D.J. Schiffrin, R. Whyman, Chem. Commun. (1994) 801802.

[32] N.G. Bastus, J. Comenge, V. Puntes, Langmuir 27 (2011) 11098-11105.

[33] N. Pazos-Perez, F.J. Garcia de Abajo, A. Fery, R.A. Alvarez-Puebla, Langmuir 28 (2012) 8909-8914.

[34] M.-S. Bernat, M.-F. Judit, G.-Q. Patricia, P.-P. Nicolas, X. Hai-nan, G.B. Neus, P. Víctor, A.A.-P. Ramon, G. Luca, J. Opt. 17 (2015) 114012.

[35] F. Benz, R. Chikkaraddy, A. Salmon, H. Ohadi, B. de Nijs, J. Mertens, C. Carnegie, R.W. Bowman, J.J. Baumberg, J. Phys. Chem. Lett. 7 (2016) 2264-2269.

[36] N.G. Bastús, F. Merkoçi, J. Piella, V. Puntes, Chem. Mater. 26 (2014) 2836-2846.

[37] M.B. Cortie, A.M. McDonagh, Chem. Rev. 111 (2011) 3713-3735.

[38] M. Mayer, L. Scarabelli, K. March, T. Altantzis, M. Tebbe, M. Kociak, S. Bals, J. Garcia de Abajo, A. Fery, L.M. Liz-Marzán, Nano Lett. 15 (2015) 5427-5437.

[39] M. Mayer, A.M. Steiner, F. Röder, P. Formanek, T. König, A. Fery, Angew. Chem. Int. Ed. advanced online publication

[40] Y. Yang, J. Liu, Z.-W. Fu, D. Qin, J. Am. Chem. Soc. 136 (2014) 8153-8156.

[41] C. Gao, Z. Lu, Y. Liu, Q. Zhang, M. Chi, Q. Cheng, Y. Yin, Angew. Chem. Int. Ed. 51 (2012) 5629-5633.

[42] J. Reguera, J. Langer, D. Jimenez de Aberasturi, L.M. Liz-Marzán, Chem. Soc. Rev. 46 (2017) 3866-3885.

[43] C. Colliex, M. Kociak, O. Stéphan, Ultramicroscopy 162 (2016) A1-A24.

[44] M. Kociak, O. Stephan, Chem. Soc. Rev. 43 (2014) 3865-3883.

[45] J. Nelayah, M. Kociak, O. Stephan, F.J. Garcia de Abajo, M. Tence, L. Henrard, D. Taverna, I. Pastoriza-Santos, L.M. Liz-Marzán, C. Colliex, Nat. Phys. 3 (2007) 348-353.

[46] A. Losquin, L.F. Zagonel, V. Myroshnychenko, B. Rodríguez-González, M. Tencé, L. Scarabelli, J. Förstner, L.M. Liz-Marzán, F.J. García de Abajo, O. Stéphan, M. Kociak, Nano Lett. 15 (2015) 1229-1237.

[47] B.S. Guiton, V. Iberi, S. Li, D.N. Leonard, C.M. Parish, P.G. Kotula, M. Varela, G.C. Schatz, S.J. Pennycook, J.P. Camden, Nano Lett. 11 (2011) 3482-3488. 
[48] L. Rodríguez-Lorenzo, R.A. Álvarez-Puebla, I. Pastoriza-Santos, S. Mazzucco, O. Stéphan, M. Kociak, L.M. Liz-Marzán, F.J. García de Abajo, J. Am. Chem. Soc. 131 (2009) 4616-4618.

[49] E.C. Le Ru, J. Grand, I. Sow, W.R.C. Somerville, P.G. Etchegoin, M. Treguer-Delapierre, G. Charron, N. Félidj, G. Lévi, J. Aubard, Nano Lett. 11 (2011) 5013-5019.

[50] W. Lu, A.K. Singh, S.A. Khan, D. Senapati, H. Yu, P.C. Ray, J. Am. Chem. Soc. 132 (2010) 18103-18114.

[51] C. Hrelescu, T.K. Sau, A.L. Rogach, F. Jäckel, J. Feldmann, Appl. Phys. Lett. 94 (2009) 153113.

[52] F. Hao, C.L. Nehl, J.H. Hafner, P. Nordlander, Nano Lett. 7 (2007) 729-732.

[53] C.G. Khoury, T. Vo-Dinh, J. Phys. Chem. C, 2008 (2008) 18849-18859.

[54] A. Guerrero-Martínez, S. Barbosa, I. Pastoriza-Santos, L.M. Liz-Marzán, Curr. Op. Colloid Interface Sci. 16 (2011) 118-127.

[55] Y. Wang, J. He, C. Liu, W.H. Chong, H. Chen, Angew. Chem. Int. Ed. 54 (2014) 20222051.

[56] X. Xia, S. Xie, M. Liu, H.-C. Peng, N. Lu, J. Wang, M.J. Kim, Y. Xia, Proc. Natl. Acad. Sci. U.S.A. 110 (2013) 6669-6673.

[57] L. Scarabelli, M. Coronado-Puchau, J.J. Giner-Casares, J. Langer, L.M. Liz-Marzán, ACS Nano 8 (2014) 5833-5842.

[58] K. Liu, Y. Bai, L. Zhang, Z. Yang, Q. Fan, H. Zheng, Y. Yin, C. Gao, Nano Lett. 16 (2016) 3675-3681.

[59] A. Genç, J. Patarroyo, J. Sancho-Parramon, G. Bastús Neus, V. Puntes, J. Arbiol, Nanophotonics, 2017, 193.

[60] M. Yang, R.A. Alvarez-Puebla, H.-S. Kim, P. Aldeanueva-Potel, L.M. Liz-Marzán, N.A. Kotov, Nano Lett. 10 (2010) 4013-4019.

[61] D.-K. Lim, K.-S. Jeon, J.-H. Hwang, H. Kim, S. Kwon, Y.D. Suh, J.-M. Nam, Nat. Nanotechnol. 6 (2011) 452-460.

[62] J.W. Kang, P.T.C. So, R.R. Dasari, D.-K. Lim, Nano Lett. 15 (2015) 1766-1772.

[63] W. Zhang, M. Rahmani, W. Niu, S. Ravaine, M. Hong, X. Lu, Sci. Rep. 5 (2015) 8382.

[64] W. Xiong, D. Sikdar, L.W. Yap, M. Premaratne, X. Li, W. Cheng, Nanoscale 7 (2015) 3445-3452.

[65] J.-M. Nam, J.-W. Oh, H. Lee, Y.D. Suh, Acc. Chem. Res. 49 (2016) 2746-2755.

[66] M.J. Schnepf, M. Mayer, C. Kuttner, M. Tebbe, D. Wolf, M. Dulle, T. Altantzis, P. Formanek, S. Forster, S. Bals, T.A.F. Konig, A. Fery, Nanoscale 9 (2017) 9376-9385.

[67] S.E. Lohse, C.J. Murphy, Chem. Mater. 25 (2013) 1250-1261.

[68] N.D. Burrows, S. Harvey, F.A. Idesis, C.J. Murphy, Langmuir 33 (2017) 1891-1907.

[69] L. Vigderman, B.P. Khanal, E.R. Zubarev, Adv. Mat. 24 (2012) 4811-4841. 
[70] D.M. Solís, J.M. Taboada, F. Obelleiro, L.M. Liz-Marzán, F.J. García de Abajo, ACS Photonics 4 (2017) 329-337.

[71] H.-Y. Chen, M.-H. Lin, C.-Y. Wang, Y.-M. Chang, S. Gwo, J. Am. Chem. Soc. 137 (2015) 13698-13705.

[72] T. Thai, Y. Zheng, S.H. Ng, S. Mudie, M. Altissimo, U. Bach, Angew. Chem. Int. Ed. 51(35) (2012) 8732-8735.

[73] A. Apte, P. Bhaskar, R. Das, S. Chaturvedi, P. Poddar, S. Kulkarni, Nano Res. 8 (2015) 907-919.

[74] M. Fan, A.G. Brolo, Phys. Chem. Chem. Phys. 11 (2009) 7381-7389.

[75] S. Guo, S. Dong, J. Mater. Chem. 21 (2011) 16704-16716.

[76] N. Vogel, M. Retsch, C.-A. Fustin, A. del Campo, U. Jonas, Chem. Rev. 115 (2015) 62656311.

[77] C. Kuttner, M. Chanana, M. Karg, A. Fery, John Wiley \& Sons, Inc.2016, pp. 159-192.

[78] N. Pazos-Perez, C.S. Wagner, J.M. Romo-Herrera, L.M. Liz-Marzán, F.J. García de Abajo, A. Wittemann, A. Fery, R.A. Alvarez-Puebla, Angew. Chem. Int. Ed. 51 (2012) 12688-12693. [79] V.V. Thacker, L.O. Herrmann, D.O. Sigle, T. Zhang, T. Liedl, J.J. Baumberg, U.F. Keyser, Nat. Commun. 5 (2014) 3448.

[80] J. Prinz, C. Heck, L. Ellerik, V. Merk, I. Bald, Nanoscale 8 (2016) 5612-5620.

[81] P. Kühler, E.-M. Roller, R. Schreiber, T. Liedl, T. Lohmüller, J. Feldmann, Nano Lett. 14 (2014) 2914-2919.

[82] C.A. Silvera Batista, R.G. Larson, N.A. Kotov, Science 350 (2015) 1242477(1-10).

[83] R. Tan, H. Zhu, C. Cao, O. Chen, Nanoscale 8 (2016) 9944-9961.

[84] T. Wang, D. LaMontagne, J. Lynch, J. Zhuang, Y.C. Cao, Chem. Soc. Rev. 42 (2013) 2804-2823.

[85] M. Schutz, S. Schlucker, Phys. Chem. Chem. Phys. 17 (2015) 24356-24360.

[86] R.P.M. Höller, M. Dulle, S. Thomä, M. Mayer, A.M. Steiner, S. Förster, A. Fery, C. Kuttner, M. Chanana, ACS Nano 10 (2016) 5740-5750.

[87] Y. Zheng, A.H. Soeriyadi, L. Rosa, S.H. Ng, U. Bach, J. Justin Gooding, Nat. Commun. 6 (2015) 8797.

[88] Y. Wang, B. Yan, L. Chen, Chem. Rev. 113 (2013) 1391-1428.

[89] L. Fabris, ChemNanoMat, 2 (2016) 249-258.

[90] Y. He, Y. Wang, X. Yang, S. Xie, R. Yuan, Y. Chai, ACS Appl. Mater. Interfaces 8 (2016) 7683-7690.

[91] A. La Porta, A. Sanchez-Iglesias, T. Altantzis, S. Bals, M. Grzelczak, L.M. Liz-Marzán, Nanoscale 7 (2015) 10377-10381.

[92] G.L. Liu, Y. Lu, J. Kim, J.C. Doll, L.P. Lee, Adv. Mat. 17 (2005) 2683-2688. 
[93] J. Henzie, S.C. Andrews, X.Y. Ling, Z. Li, P. Yang, Proc. Natl. Acad. Sci. U.S.A. 110 (2013) 6640-6645.

[94] J. Gong, R.S. Newman, M. Engel, M. Zhao, F. Bian, S.C. Glotzer, Z. Tang, Nat. Commun. 8 (2017) 14038.

[95] D.A. Walker, K.P. Browne, B. Kowalczyk, B.A. Grzybowski, Angew. Chem. Int. Ed. 49 (2010) 6760-6763.

[96] L. Scarabelli, C. Hamon, L.M. Liz-Marzán, Chem. Mater. 29 (2017) 15-25.

[97] C. Hamon, S.M. Novikov, L. Scarabelli, D.M. Solís, T. Altantzis, S. Bals, J.M. Taboada, F. Obelleiro, L.M. Liz-Marzán, ACS Photonics 2 (2015) 1482-1488.

[98] P.L. Stiles, J. A. Dieringer, N. C. Shah, R. P. Van Duyne, Annu. Rev. Anal. Chem. 1 (2008) 601-626.

[99] S.A. Meyer, E.C.L. Ru, P.G. Etchegoin, J. Phys. Chem. A 114 (2010) 5515-5519.

[100] A. P. Craig, A. S. Franca, J. Irudayaraj, Annu. Rev. Food Sci. Technol. 4 (2013) 369-380.

[101] A. Jaworska, S. Fornasaro, V. Sergo, A. Bonifacio, Biosensors 6 (2016) 47.

[102] F. Casadio, M. Leona, J.R. Lombardi, R. Van Duyne, Acc. Chem. Res. 43 (2010) 782791.

[103] R.A. Alvarez-Puebla, L.M. Liz-Marzán, Angew. Chem. Int. Ed. 51 (2012) 11214-11223.

[104] S.J. Lee, M. Moskovits, Nano Lett. 11 (2011) 145-150.

[105] L. Guerrini, I. Rodriguez-Loureiro, M.A. Correa-Duarte, Y.H. Lee, X.Y. Ling, F.J. Garcia de Abajo, R.A. Alvarez-Puebla, Nanoscale 6 (2014) 8368-8375.

[106] D. Tsoutsi, J.M. Montenegro, F. Dommershausen, U. Koert, L.M. Liz-Marzán, W.J. Parak, R.A. Alvarez-Puebla, ACS Nano 5 (2011) 7539-7546.

[107] L.A. Lane, X. Qian, S. Nie, Chem. Rev. 115 (2015) 10489-10529.

[108] V. Templier, A. Roux, Y. Roupioz, T. Livache, Trends Anal. Chem. 79 (2016) 71-79.

[109] T. Gong, Z.-Y. Hong, C.-H. Chen, C.-Y. Tsai, L.-D. Liao, K.V. Kong, ACS Nano 11 (2017) 3365-3375.

[110] C.L. Zavaleta, B.R. Smith, I. Walton, W. Doering, G. Davis, B. Shojaei, M.J. Natan, S.S. Gambhir, Proc. Natl. Acad. Sci. U.S.A. 106 (2009) 13511-13516.

[111] J.-F. Li, Y.-J. Zhang, S.-Y. Ding, R. Panneerselvam, Z.-Q. Tian, Chem. Rev. 117 (2017) 5002-5069.

[112] M.B. Wabuyele, F. Yan, G.D. Griffin, T. Vo-Dinh, Rev. Sci. Instrum. 76 (2005) 063710.

[113] J. Kneipp, H. Kneipp, W.L. Rice, K. Kneipp, Anal. Chem. 77 (2005) 2381-2385.

[114] A. Matschulat, D. Drescher, J. Kneipp, ACS Nano 4 (2010) 3259-3269.

[115] C.E. Talley, L. Jusinski, C.W. Hollars, S.M. Lane, T. Huser, Anal. Chem. 76(23) (2004) 7064-7068.

[116] R.A. Alvarez-Puebla, E. Arceo, P.J.G. Goulet, J.J. Garrido, R.F. Aroca, J. Phys. Chem. B 109 (2005) 3787-3792. 
[117] R.F. Aroca, R.A. Alvarez-Puebla, N. Pieczonka, S. Sanchez-Cortez, J.V. Garcia-Ramos, Adv. Colloid Interface Sci. 116 (2005) 45-61.

[118] Q. Yu, G. Golden, Langmuir 23 (2007) 8659-8662.

[119] C. Hamon, M.N. Sanz-Ortiz, E. Modin, E.H. Hill, L. Scarabelli, A. Chuvilin, L.M. LizMarzán, Nanoscale 8 (2016) 7914-7922.

[120] A. Carretero-Genevrier, G.L. Drisko, D. Grosso, C. Boissiere, C. Sanchez, Nanoscale 6 (2014) 14025-14043.

[121] C. Sanchez, C. Boissière, D. Grosso, C. Laberty, L. Nicole, Chem. Mater. 20 (2008) 682737.

[122] F. Sun, H.-C. Hung, A. Sinclair, P. Zhang, T. Bai, D.D. Galvan, P. Jain, B. Li, S. Jiang, Q. Yu, Nat. Commun. 7 (2016) 13437.

[123] G.M. Whitesides, Lab Chip 13 (2013) 11-13.

[124] C. Hamon, L.M. Liz-Marzán, Chem. Eur. J. 21 (2015) 9956-9963.

[125] Y. Xia, D. Qin, G. M Whitesides, Nat. Protocols 5 (2010) 491-502.

[126] V. Flauraud, M. Mastrangeli, G.D. Bernasconi, J. Butet, D.T.L. Alexander, E. Shahrabi, O.J.F. Martin, J. Brugger, Nat. Nanotechnol. 12 (2016) 73-80.

[127] C. Hamon, S. Novikov, L. Scarabelli, L. Basabe-Desmonts, L.M. Liz-Marzán, ACS Nano 8 (2014) 10694-10703.

[128] C. Hamon, M. Postic, E. Mazari, T. Bizien, C. Dupuis, P. Even-Hernandez, A. Jimenez, L. Courbin, C. Gosse, F. Artzner, V. Marchi-Artzner, ACS Nano 6 (2012) 4137-4146.

[129] F. De Angelis, F. Gentile, F. Mecarini, G. Das, M. Moretti, P. Candeloro, $\quad$ M. $\quad$ L. Coluccio, G. Cojoc, A. Accardo, C. Liberale, R. P. Zaccaria, G. Perozziello, L. Tirinato, A.

Toma, G. Cuda, R. Cingolani, E. Di Fabrizio, Nat. Photonics 5 (2011) 682-687.

[130] L. Polavarapu, A.L. Porta, S.M. Novikov, M. Coronado-Puchau, L.M. Liz-Marzán, Small 10 (2014) 3065-3071.

[131] A. Abou-Hassan, O. Sandre, V. Cabuil, Angew. Chem. Int. Ed. 49 (2010) 6268-6286.

[132] A.F. Chrimes, K. Khoshmanesh, P.R. Stoddart, A. Mitchell, K. Kalantar-zadeh, Chem. Soc. Rev. 42 (2013) 5880-5906.

[133] D. Lee, S. Lee, G.H. Seong, J. Choo, E.K. Lee, D.-G. Gweon, S. Lee, Appl. Spectrosc. 60 (2006) 373-377.

[134] A. Walter, A. Marz, W. Schumacher, P. Rosch, J. Popp, Lab Chip 11 (2011) 1013-1021.

[135] S. Begolo, D.V. Zhukov, D.A. Selck, L. Li, R.F. Ismagilov, Lab Chip 14 (2014) 46164628.

[136] L. Xu, H. Lee, D. Jetta, K.W. Oh, Lab Chip 15 (2015) 3962-3979.

[137] I.K. Dimov, L. Basabe-Desmonts, J.L. Garcia-Cordero, B.M. Ross, A.J. Ricco, L.P. Lee, Lab Chip 11 (2011) 845-850. 